\title{
Processamento Visual da Forma: Análise de Sistema Linear e alguns Paradigmas Psicofísicos
}

\author{
NatanaelAntoniodosSantos ${ }^{2}$ \\ Maria Lúcia deBustamanteSmas ${ }^{3}$ \\ UniversidadeFederaldePemambuco
}

\begin{abstract}
Resumo
O objetivo deste trabalho é discutir alguns aspectos conceituais básicos da análise de Fourier enquanto ferramenta que fundamenta a perspectiva de filtros ou canais múltiplos de freqüências espaciais no estudo do processamento visual da forma. Serão também discutidos alguns dos principais paradigmas psicofísicos utilizados para caractenzar a resposta do sistema visual humano para filtros de freqüências espaciais de banda estreita. A análise de sistema lineare alguns paradigmas psicofísicos têm contribuído para o desenvolvimento teónico da percepção e do processamento visual da forma.

Palavras-chave: Processamento visual de forma; análise de Founier, paradigma psicofísico; filtro de freqüência espacial; freqüência espacial.
\end{abstract}

\section{Visual Processing of Form: Linear System Analysis and Some Psychophysical Paradigms}

\begin{abstract}
The goal of this work is to discuss some basic aspects of Fourier analysis as a tool to be used in the approach of multiple channels of spatial frequencies on the study of visual processing of form. Some of the psychophysical paradigms more frequently used to characterize response of the human visual system to spatial frequency filter of narrow-band. The linear system analysis and some psychophysical paradigms have contributed to theoretical development of perception and of the visual processing of form.

Keywords: Visual processing of form; Fourier analysis; psychophysical paradigm; spatial filter; spatial frequency.
\end{abstract}

A análise de sistema linear é fundamentada em um dos teoremas matemáticos de Fourier. Este afirma, em essência, que qualquerestímulo contínuo e periódico pode ser representado pela soma de uma série de ondas senoidais simples com comprimento de onda, fase e amplitude apropriados. Posteniormente, verificou-se que estímulo não periódico também poderia ser descrito como a soma de ondas senos e/ou cossenos no caso em que todos os componentes de freqüência fossem incluídos. Assim, em outras palavras, estímulo periódico tem espectro discreto, enquanto o estámulo não periódico tem espectro contínuo.

A análise de Fourier tem sido aplicada em vánias áreas da ciência com propósito diversificado. Por exemplo, no século XIX, Ohm e Helmholtz utilizaram a análise de Fourier como base para estudar o sistema auditivo (Helmholt, 1877; Ohm, 1843). Posteriormente, já no século XX, a análise de sistema linear migrou do sistema

${ }^{1}$ Endereço para correspondência: Rua Manoel de Macedo, s/n, 401, Ed. Camburiú, 58052-290. João Pessoa, PB. Fone: (81) 32713734, Fax: (81) 32718271. E-mail: natanael@npd.ufpe.br

${ }^{2}$ Bolsa Pesquisador DCR/CNPq 300746/99-7.

${ }^{3}$ Bolsa Pesquisador CNPq 523792/95-0 auditivo para o sistema visual, onde foi utilizada para estudar o sistema visual através da perspectiva de canal simples (de Lange, 1952, 1957; Ives, 1922; Schade, 1948, 1956; Selwyn, 1948). Em seguida, também foi utilizada para estudar o sistema visual dentro da abordagem de canais múltiplos (Blakemore \& Campbell, 1969a, 1969b; Campbell \& Robson, 1968; Sachs, Nachmias \& Robson, 1971). De acordo com a perspectiva de canais múltiplos, o sistema visual processa freqüência espacial de maneira análoga à forma que o sistema auditivo processa freqüência temporal, isto é, uma espécie de análise de Fourier (Blakemore \& Campbell, 1969b). Para maiores informações sobre a idéia de sistema lineares em visão e o modelo de canais múltiplos ver, porexemplo, Comsweet (1970), Sekuler (1974), R. L De Valois e K. K. De Valois (1988), Santos (1999) e Santos e Simas (2001).

R. L. De Valois e K. K. De Valois (1988), Santos (1999) e Santos e Simas (2001) discutem a aplicação da análise de sistema linear no processamento visual de padrões tanto do ponto de vista local (ponto porponto) como global (área inteira). Esse manuscrito enfatiza o processamento visual de áreas ou faixas inteiras do campo visual, isto é, processamento global. 


\section{Análise de Fourier e Filtros Múltiplos de Freqüências Espaciais}

Os estudos que procuram caracterizar a resposta do sistema visual, em relação à percepção e processamento visual da forma, geralmente utilizam ondas simples que possuem freqüências espaciais relacionadas através de razões matemáticas simples, de forma que os hamônicos ou freqüências mais altas são múltiplos inteiros da freqüência mais baixa ou fundamental. Por exemplo, uma onda quadrada de frequiência fe amplitude um (1) pode ser analisada através da soma de ondas senoidais, que são os harmônicos ímpares inteiros de f, representado por (Comsweet, 1970; R. L De Valois \& K. K. De Valois, 1988):

\section{$4 / p[\operatorname{sen}(f)+\operatorname{sen}(3 f) / 3+\operatorname{sen}(5 f) / 5+\operatorname{sen}(7 f) / 7+\operatorname{sen}(9 f) /$ $9+\ldots+\operatorname{sen}(n f) / n]$.}

Em outras palavras, a equação acima mostra que 0 espectro de Founier de uma onda quadrada contém todos os harmônicos ímpares da freqüência fundamental f, com amplitude inversamente proporcional aos números dos hamônicos (Nachmias, Sansbury, Vassilev \&Weber, 1973).

Neste contexto, emergem dois conceitos constituídos pelos processos de análise e síntese que fundamentam 0 processamento da informação e da percepção visual da forma dentro da abordagem de canais múltiplos de freqüências espaciais. Considerando que a informação que chega ao sistema pode ser decomposta em um primeiro estágio para ser composta posteriomente em um outro estágio, a análise e a síntese permitem prever a resposta do sistema visual humano (SVH) para qualquer distribuição de luminância. Logo, análise ou transfomada de Founieré a decomposição de qualquer forma de onda arbitránia em seus componentes elementares que nesta teoria seriam descritos em temos de ondas senoidais e/ ou cossenoidais, enquanto a síntese ou transformada inversa é a composição de qualquer est́mulo complexo ou onda arbitrária pela soma de ondas senoidais e/ou cossenoidais (Comsweet, 1970). Este é, por exemplo, o princípio de sintetizadores que simulam instrumentos musicais.

Um estímulo descrito no domínio da freqüência só é completo se ele incluir informações sobre amplitude, freqüência e fase. Neste sentido, denomina-se de espectro a uma descrição destas que leva em consideração a resolução completa do estímulo original. Assim, porexemplo, em uma filtragem unidirecional do campo visual inteiro, a análise ou transformada de Fourier leva em consideração a freqüência espacial, a amplitude (ou contraste) e a fase espacial. Enquanto, em uma filtragem bidimensional, a análise ou transformada de Fourier leva em consideração a onientação espacial, além da freqüência espacial, da amplitude e da fase do padrão. Neste contexto, estímulos do tipo funções senoidais ou cossenoidais são padrões elementares descritos na literatura em termos de sua freqüência espacial.

Freqüência espacial é definida como o número de ciclos (ou peńodos) pormedida de espaço. Por exemplo, no caso de uma grade senoidal, um ciclo corresponde a uma listra clara e uma escura. Em processamento visual da forma, a medida de espaço que define a freqüência foi convencionalmente denominada de grau de ângulo visual ou ciclo porgrau de ângulo visual (cpg). Freqüências de 1 e 4 cpg, por exemplo, teriam um e quatro ciclos por grau de ângulo visual, respectivamente. Amplitude é a distância entre o pico e o vale da onda dividido pordois. Fase é a posição da onda ou da grade senoidal no espaço. Grade senoidal é um estímulo cuja luminância varia senoidalmente em uma direção do espaço e em um sistema de coordenadas cartesianas.

\section{Sistema Linear e Sistema Visual}

A maneira mais simples de combinarinfomação é atravésde um modelo lineare a linearidade prediz que a resposta do sistema visual para a soma de dois estímulos componentes será a soma das respostas para os estímulos componentes apresentadosindividualmente. (Olds \& Engel, 1998, p. 2109)

Um sistema é dito linear quando os componentes de Fourier transferidos pelo sistema visual se adicionam algebricamente no plano da imagem, isto é, obedecem ao princípio de superposição. Isto significa que a resposta é proporcional ao estímulo, ou seja, aumentando-se a intensidade de luminância, aumenta-se a intensidade da imagem na mesma proporção. Formalmente, um sistema obedece ao princípio de superposição se, por exemplo, a resposta do sistema aos estímulos fe(x) $+2 \mathrm{fe}(\mathrm{x})$ é igual $\mathrm{fs}(\mathrm{x})+2 \mathrm{fs}(\mathrm{x})$, onde e e s subscrevem a função de "entrada" e "saída", respectivamente, no domínio do espaço (f corresponde a freqüência espacial).

Neste sentido, pode-se questionar alguns aspectos da análise de Founer e filtragem espacial global: 1) a análise de sistema linear só permite prever a saída corretamente quando aplicado a sistemas estritamente lineares ou a sistemas estritamente não lineares capazes de operar linearmente em uma determinada faixa (Comsweet, 1970; Santos, 1999; Santos \& Simas, 2001). De fato, um sistema que tem limiares de ativação não é linear, mas pode ser considerado linear em torno de seu limiar de ativação (Comsweet, 1970). 2) Invariância ou homogeneidade, ou 
seja, um sistema só pode ser considerado invariante quando a função de sensibilidade ao contraste (FSC) é a mesma em qualquer ponto do sistema, no qual incide a luminância do objeto ou quando apresenta a mesma curva de resposta independente do tempo em que foi medida. Apesar da heterogeneidade da retina, esta questão pode ser resolvida se delimitara área da retina à região da fóvea e parafóvea. 3) Isotropia do sistema, isto é, o sistema só é considerado isotrópico quando a FSC é semelhante, independentemente da orientação da imagem (vertical, horizontal ou oblíquo) em relação ao eixo óptico. Para maiores informações sobre estes aspectos ver, por exemplo, Comsweet (1970) e Sekuler (1974).

Em 1964, Spening comentou que a resposta espacial do sistema visual pode ser considerada linear e invariante se a resposta do sistema para uma freqüência do estímulo que entra (grade senoidal) for igual à que sai (grade senoidal) mesmo, porém, que a amplitude ou a fase seja diferente, ou até mesmo ambas.

A FSC ou a curva de limiar de contraste (1/FSC) é uma função que geralmente estima a visibilidade de grades ou qualquerpadrão como uma função de sua freqüência espacial. A FSC, que geralmente é mensurada com paradigmas psicofísicos, representa um envelope de sensibilidade para a sénie total de canais ou filtros, cada um sensível a uma faixa restrita e discreta do espectro de freqüência espacial (Graham \& Nachmias, 1971; Sachs \& cols., 1971; Sekuler, 1974).

\section{Linearidade de Contraste}

O resultado de como a percepção de contraste está relacionada ao contraste físico real do estímulo foi o objeto de muito estudo nas investigações eletrofisiológicas (Campbell \& Maffei, 1970), fisiológicas (Maffei \& Fiorentini, 1973) e psicofísicas (Georgeson \& Sullivan, 1975; Ginsburg, Cannon \& Nelson, 1980; Kulikowski \& Gorea, 1978; Legge, 1981). Os estudos eletrofisiológicos e fisiológicos de Campbell, Maffei e Fiorentini demonstraram que a percepção de contraste parece ser linearmente relacionada ao logaritmo do contraste do estímulo.

Linearidade de contraste pode serinvestigada de duas maneiras diferentes, diretamente ou indiretamente. Exemplos de avaliações diretas podem ser vistas no estudo eletrofisiológico de Campbell e Maffei (1970) e no estudo de estimação de magnitude de Ginsburg e colaboradores (1980). Técnica eletrofisiológica testa a lineanidade de contraste medindo a amplitude do potencial evocado visual (VEP) sustentado com o contraste físico do estímulo. $O$ fato de que a amplitude varia linearmente com o logaritmo do contraste implica em uma relação direta entre o VEP mensurado e o contraste do estímulo. Por outro lado, na técnica psicofísica de estimação de magnitude, os voluntánios designam valores subjetivos para parear o contraste de um estúmulo alvo. Nesse caso, os achados parecem mostrar que o logaritmo dos valores atribuídos à percepção de contraste está linearmente relacionado ao logaritmo de contraste físico.

Uma avaliação indireta de linearidade de contraste é feita quando as funções de respostas diferentes que caractenzam um filtro em particular são usadas para predizer umaà outra com base na teonia de sistemas lineares. Nesse caso, o poder preditivo depende da lineanidade ao contraste existirou não.

Demonstração de linearidade e invariância no espaço para filtros de bandas estreitas não é, em si, prova que qualquer transformada de Fourier discreta esteja sendo feita no SVH. Entretanto, seria um pré-requisito para tal processo acontecer.

\section{Métodos Psicofísicos}

Os paradigmas psicofísicos geralmente procuram encontrar relações existentes entre as caracteństicas físicas do estímulo e suas características perceptuais. Em percepção e processamento visual da forma, por exemplo, uma característica física seria a modulação de luminância enquanto a característica perceptual seria o contraste. A modulação de luminância ocorre em tomo de uma luminância média cujo correlato perceptual é o brilho. Neste sentido, o contraste permite que se perceba a diferença entre modulações de luminâncias diferentes (estímulos de freqüências espaciais diferentes), pois a sensibilidade ao contraste está relacionada à diferença existente entre picos e depressões das ondas senoidias ou cossenoidais. Essa diferença, por exemplo, traduz o valor de contraste requerido pelo SVH para distinguir um estímulo de freqüência espacial de outras freqüências ou de um campo uniforme de luminância média. É desta maneira que se mensura a função de sensibilidade ao contraste ou curvas de resposta ao contraste para filtros de freqüências espaciais. Essas são, por exemplo, estimativas clássicas em percepção e processamento visual da forma. A seguir serão citados alguns paradigmas psicofísicos utilizados para estimarfiltrosespaciais ou canais de freqüências espaciais de banda estreita.

Um canal ou um filtro corresponde a uma estrutura independente no sistema que lida com uma faixa discreta e estreita de freqüência espacial (Santos \& Simas, 2001), ou à população de neurônios envolvidas no processamento seletivo de freqüências espaciais (Santos, 1999).

Contraste é a relação entre a luminância máxima e luminância mínima, representado matematicamente da seguinte forma: 


$$
\mathrm{C}=\mathrm{L}_{\max }-\mathrm{L}_{\min } / \mathrm{L}_{\max }+\mathrm{L}_{\min }
$$

Quando a modulação do contraste é senoidal ou cossenoidal, trata-se de um estímulo considerado elementar para uma análise de Founier.

\section{Métodos Psicofísicos e Filtro Espacial de Banda Estreita}

Os estudos psicofísicos e fisiológicos que utilizaram a análise de sistemas lineares (análise de Fourier discreta) para investigar a hipótese de filtragem de freqüências espaciais em faixas distintas, estreitas e parcialmente superpostas usaram fenômenos visuais ou paradigmas tais como adaptação, mascaramento, somação de resposta de sub-limiares e somação de resposta de supra-limiar.

\section{Paradigmadeadaptação}

O fenômeno de adaptação à freqüência espacial está relacionado à perda temporária na sensibilidade para detectaruma grade de freqüência espacial de teste, quando esta se segue à exposição prolongada a uma outra grade de alto contraste. $\mathrm{O}$ efeito da adaptação geralmente é máximo quando a grade de teste e a grade à qual o sistema visual se adaptou têm onientações espaciais e freqüências espaciais idênticas, que pode implicar que os dois estímulos são processados pelo mesmo canal. Por outro lado, o efeito da adaptação diminui à medida que a freqüência espacial ou a orientação espacial da grade de teste se afasta da freqüência ou da orientação da grade adaptada, respectivamente. Logo, se as freqüências dos dois estímulos são suficientemente diferentes, elas são processadas por canais separados de freqüências espaciais. Esse efeito de adaptação, relatado primeiro porGilinski (1968) para orientação, foi usado para derivar funções de resposta à freqüência para filtros de freqüência espacial de banda estreita com pico de sensibilidade em diferentes faixas (Blakemore \& Campbell, 1969a, 1969b; Graham, 1972; Tolhurst \& Barfield, 1978). Blakemore, Campbell e Graham também usaram um método de ajustamento para estimar os efeitos da elevação do limiar de contraste nas vánias freqüências adaptadas (Blakemore \& Campbell, 1969a, 1969b; Graham, 1972).

Adaptação à freqüência espacial específica independe tanto da adaptação local na retina (Jones \& TulunayKeesey, 1975) como do efeito de pós-imagens (Smith, 1977). A adaptação tem um efeito prolongado se, logo depois do período de adaptação inicial de pelo menos um minuto, períodos de adaptação subseqüentes que durem de 15-45s são interpostos a cada teste de 10 s a 20s de duração (Bodinger, 1978; Campbell, Carpenter \& Levinson, 1969a; Campbell, Cooper \& Enroth-Cugell, 1969b). Uma base biológica para tal seqüência de adaptação específica foi mostrada por Maffei e colaboradores nas células corticais simples (Maffei, Fiorentini \& Bisti, 1973).

O paradigma de adaptação continua sendo uma técnica muito utilizada para estudaros mecanismos sintonizados para freqüências espaciais e canais seletivos para orientação espacial (Greenlee \& Magnussen, 1998; Howard, Tregear \&Wemer, 2000; Humanski \& Wilson, 1993; Issa, Trepel \& Stryker, 2000; Kortum \& Geisler, 1995; Meese \&Georgeson, 1996; Menees, 1998; Snowden \& Hammett, 1996; Stromeyer, Klein, Dawson \& Spillmann, 1982; Wainwright, 1999).

\section{Paradigmademascaramento}

Este paradigma comportamental tem sido muito utilizado na pesquisa visual em vánias abordagens diferentes (Ascher \& Grzywacz, 2000; Bennett\& Cortese, 1996; Bowen \&Wilson, 1994; Breitmeyer \& Ganz, 1976; Dorais \& Sagi, 1997; Mansfield \& Parker, 1993; Mussap \& Levi, 1997; Olds \& Engel, 1998; Polat \& Sagi, 1993; Stromeyer \& Julesz, 1972; Stromeyer \& cols., 1982; Wilson, McFarlane \& Phillips, 1983; Wilson, Nagy, Mets \& Perrera, 1988; Yang \& Stevenson, 1999). O fenômeno básico envolve a diminuição temporána da sensibilidade para uma determinada classe de estímulos de testes. Esta diminuição acontece quando outros estímulos que funcionam como máscaras (semelhantes ou idênticose queinduzem maiorsensibilidade) são apresentadossimultaneamente ou deslocados no tempo pornão mais que $300 \mathrm{~ms}$. O estímulo que age como máscara pode preceder, acompanharou sersubseqüente ao estímulo de teste.

Na estimação da largura de banda de um filtro de freqüência espacial, o paradigma consiste em uma grade de contraste alto de freqüência espacial específica apresentada em breve período de tempo; por exemplo, 20 ms antes, após ou simultaneamente à apresentação de uma grade de teste de contraste mais baixo. A freqüência espacial de teste pode seridêntica ou não àquela freqüência da grade que está sendo utilizada como máscara. A quantidade de contraste requerida para apenas detectara grade de teste na presença de cada freqüência espacial utilizada como máscara é considerada proporcional às amplitudes que definem a banda do filtro (Julesz, 1980; Legge, 1978). A interpretação dada a este paradigma é semelhante à interpretação dada ao paradigma de adaptação. Ou seja, se uma grade afeta a detecção de outra de freqüência semelhante é porque elas estão possivelmente interagindo no mesmo canal, assim como os efeitos são mais intensos quando o estúmulo de máscara e de teste têm a mesma freqüência espacial e orientação (Bowen \& Wilson, 1994). 
Paradigma desomaçãoderesposta desub-limiares

Somação de resposta de sub-limiares é uma técnica na qual dois estímulos são apresentados simultaneamente, ambos com valores de contrastes abaixo do nível requerido para detecção independente (i.e., contraste abaixo dos valores de limiar). Se o mesmo mecanismo é utilizado para detectar ambos os estímulos, isto é, se a detecção de qualquer dos estímulos depende do mesmo mecanismo, então os níveis de excitação se somam e o limiar de contraste pode ser atingido. King-Smith e Kulikowisk relataram expenimentos com esse paradigma (King-Smith \& Kulikowski, 1975; Kulikowski \& KingSmith, 1973). Recentemente, o paradigma de somação de sub-limiar tem sido utilizado para analisar interações de mecanismos visuais espaciais que respondem para cores (Garcia, Nieves, Valero \& Romero, 2000).

A lógica do paradigma de somação de resposta de sublimiares é que, se dois padrões são apresentados simultaneamente com contrastes em níveis sub-limiares, esses podem deixar de ser percebidos exceto se o mecanismo detector de ambos for coincidente. Nesse caso, se os dois estímulos são processados por um canal comum, a combinação entre eles pode exceder o limiar de deteç̧ão. E se eles são processados por canais diferentes, a detecção acontece de forma independente e não há somação entre eles. Assim, não há somação e nem a percepção do estímulo porque o limiarde deteç̧ão não foi atingido.

\section{Paradigmadesomaçãoderesposta desupra-imiar}

Este método é uma adaptação do paradigmas de sublimiarmencionado acima. A diferença básica é que invés de utilizar níveis de contraste sub-limiarpara mensuraro máximo de uma função, utiliza-se somação de resposta de supra-limiar, onde a freqüência de teste é somada ponto a ponto à freqüência de fundo que é apresentada em nível de contraste supra-limiar.

Neste, enquanto o contraste da freqüência de teste é variado de acordo com a sensibilidade do voluntário, o contraste da freqüência de fundo é fixado acima do limiar (i.e., supra-limiar). Desta forma, se um estímulo contendo apenas uma das freqüências de fundo é comparado a um estímulo em que a mesma freqüência de fundo é somada à freqüência de teste, a única maneira de diferenciarentre os dois estímulos é detectara presença da freqüência de teste em um deles. Simas e Santos relataram expenimentos com esse paradigma (Santos, 1996, 1999; Simas, 1985; Simas \& Dodwell, 1990; Simas, Frutuoso \& Vieira, 1992; Simas \& Santos, 1998).

No caso do paradigma de resposta de supra-limiar, três tipos de respostas podem ocomerdurante o processo de filtragem: 1) aquelas em que determinadas freqüências de fundo podem se somarà freqüência de teste do filtro facilitando a sua detecção (efeito de somação); 2) aquelas em que as freqüências de fundo e de teste podem não se somar, não facilitando nem dificultando sua detecção (efeito independente); ou, 3) aquelas em que as freqüências de fundo podem inibir a percepção da freqüência de teste do filtro, dificultando a sua detecção (efeito inibitónio). A sensibilidade ao contraste da freqüência de teste em função das freqüências de fundo, usando como referência o limiar absoluto de detecção da freqüência de teste, indicará a independência dos mecanismos de detecção ou os efeitos de somação ou inibição.

A interpretação que Santos (1999) e Simase Santos (1998) têm dado ao paradigma de resposta de supra-limiaré: 1) se a superposição da freqüência de teste do filtro à freqüência de fundo facilita ou diminui o valor de contraste absoluto necessário à detecção pode ocomer que a freqüência de teste e a freqüência de fundo estão sendo processadas pelo mesmo mecanismo ou canal; 2) se a superposição da freqüência de testeà freqüência de fundo dificulta ou aumenta o valor de contraste absoluto, elas podem serprocessadas porcanais distintos que interagem entre si possivelmente de forma antagônica; 3) se a superposição da freqüência de teste à freqüência de fundo não facilita e nem dificulta 0 valorde contrasteabsoluto (efeito independente), a freqüência de teste e a freqüência de fundo possivelmente estão sendo processadas porcanais distintos que não interagem entre si.

\section{Aspectos Gerais da Estimativa da Largura de Banda de Filtros de Freqüências Espaciais}

Cada uma das técnicas psicofísicas ou comportamentais já imaginadas para se estimar a largura de banda de um filtro foi bem sucedida. Entretanto, elas necessaniamente não geraram resultados totalmente semelhantes, pois existem dificuldades com a variabilidade na obtenção de estimativas da largura de banda de um filtro no domínio da freqüência. As propriedades dos canais dependem do método empregado (Billock \& Harding, 1996), logo dependendo da técnica utilizada podem-se obtervalores diferentes. Por exemplo, experimentos com adaptação sugerem canais mais amplos do que com somação de resposta de sub-limiares (Wilson \&Wilkinson, 1997).

Entende-se que qualquer método simples pode ter limitações, até porque o mesmo depende de interações específicas. É possível que cada paradigma, pelo menos em parte, envolva estruturas diferentes dentro das opções de síntese e análise pemitida pelo sistema visual humano. A especificidade de cada canal revelada por um método simples pode ser de fato uma interação particular para aquele paradigma. Na verdade, cada uma dessas técnicas envolve aceitações implícitas sobre interações espaço- 
temporais, que são diferentes em cada caso (ver discussão em Graham, 1980). Entretanto, resultados diferentes na faixa de sintonia dos canais não invalidam os modelos de canais múltiplos.

A idéia geral é que freqüência espacial e orientação são processadas por canais interdependentes e seletivos para \pm 1 oitava de freqüência espacial e para \pm 15 graus para orientação (Blakemore \& Campbell, 1969a; Blakemore \& Campbell, 1969b; Campbell \& Maffei, 1970; Carrasco, Mclean, Katz \& Frieder, 1998; Sekuler, 1974). Assim, as freqüências características dos canais seletivos a freqüências espaciais na visão central humana cobrem uma faixa de 3-4 oitavas (Blakemore \& Campbell, 1969b; Campbell, Cooper, Robson \& Sachs, 1969c). A largura da banda é sempre medida em oitavas, ou seja, uma curva que mostra variação de sensibilidade entre 8 e $32 \mathrm{cpg}$ tem uma largura de banda de duas oitavas. Uma oitava é uma alteração na freqüência por um fator de dois. Em outras palavras, oitavas de freqüência espacial são incrementos iguais em uma escala logańtmica (Blakemore, Nachmias \& Sutton, 1970).

O tamanho da imagem visual também pode influenciar as funções de resposta à freqüência espacial. Estúmulos de freqüências espaciais baixas (truncados) podem resultarem uma "não observação" da periodicidade do estímulo, por exemplo, se a imagem exibe menos do que três ciclos completos de grades senoidais (Legge, 1978).

Em resumo, teoricamente a largura de banda de um filtro se refere à função de resposta para as freqüências espaciais desse filtro. Dentro desse contexto, espera-se que a curva de resposta para um filtro transmita informações (1) sobre a banda (ou bandas) de passagem do filtro, (2) sobre as freqüências que o filtro responde menos ou não responde (deixa passar alguma coisa ou não deixa passar nada), e (3) sobre aquelas freqüências que estão fora da faixa de resposta do filtro. Éimportante destacar que, quanto mais estreita a largura da banda, mais alta sua seletividade à freqüência espacial. $O$ conjunto de respostas visuais obtido é então tomado como uma estimativa da função de sensibilidade ao contraste de freqüências espaciais do filtro.

\section{Considerações Finais}

A análise de Fourier é uma abordagem sólida, clara, pode ser empregada em qualquer situação real e pode fazerpredições quantitativas, isto é, ela pode especificar combinações de um número finito de componentes elementares dependendo da precisão exigida sendo essencial para padrões de freqüências espaciais com alta periodicidade. Neste sentido, a análise de sistema linear em princípio é ideal para o processamento de áreas ou faixas inteiras do campo visual (processo global), pois a amplitude de Founier para uma determinada freqüência espacial e onientação é deteminada pela forma de onda naquela onientação estendida infinitamente no espaço. É dentro deste contexto que o processo de análise e síntese forma parte da base matemática que fundamenta a perspectiva teónica de canais múltiplos de freqüências espaciais, pois de acordo com esta perspectiva, o sistema visual decompõe o estímulo complexo (ou uma cena visual) em seus componentes elementares (ondas senoidais ou cossenoidais), um tipo de análise de Fourier. Desta maneira, as freqüências espaciais seriam os atributos ou as subunidades nas quais um padrão complexo (ou cena visual) senia decomposto, processado e percebido pelo sistema visual.

Em resumo, a importância desse modelo reside no fato de que as respostas para estímulos visuais complexos podem ser preditas a partir de respostas para estímulos com freqüências espaciais elementares através do uso da séne de Founier.

Os paradigmas psicofísicos mencionados acima têm sido importantes para caracterizar a curva de resposta ao contraste do sistema visual humano para padrões de freqüências espaciais e têm contribuído para consolidara perspectiva de canais múltiplos, isto é, a existência de filtros de freqüência espacial de banda estreita.

Assim, a análise de Fourier e alguns paradigmas psicofísicos têm contribuído para o estudo e desenvolvimento teónico das pesquisas e é dentro deste contexto que temos procurado fundamentar parte da base teórica de nossos estudos sobre processamento visual da forma.

\section{Referências}

Ascher, D. \& Grzywacz, N. M. (2000). A Bayesian model of temporal frequency masking. Vision Research, 40(16), 2219-2232.

Bennett, P. J. \& Cortese, F. (1996). Masking of spatial frequency in visual memory depends on distal, not retinal, frequency. Vision Research, 36, 233-238.

Billock, V. \& Harding, T. (1996). Evidence of spatial and temporal channels in the correlational structure of human spatial temporal contrast sensitivity. Journal of Physiology, 490(2), 509-517.

Blakemore, C. \& Campbell, F. C. (1969a). Adaptation to spatial stimuli. Journal of Physiology, 200, 11-12p.

Blakemore, C. \& Campbell, F. C. (1969b). On the existence of neurons in the human visual system selectively sensitive to the orientation and size of retinal images. Journal of Physiology, 203, 237-260.

Blakemore, C., Nachmias, J. \& Sutton, P. (1970). The perceived spatial frequency selective neurones in the human brain. Journal of Physiology, 210, 727-750.

Bodinger, D. M. (1978). The decay of grating adaptation. Vision Research, $18,89-91$. 
Bowen, R. W. \& Wilson, H. R. (1994). A two-process analysis of pattem masking. Vision Research, 34, 645-657.

Breitmeyer, B. G. \& Ganz, L (1976). Implications of sustained and transient channels for theories of visual pattern masking, saccadic suppression, and information processing. Psychological Review, 83, 1-36.

Campbell, F. W., Carpenter, R. H. S. \& Levinson, J. Z. (1969a). Visibility of aperiodic pattems compared with that of sinusoidal gratings. Journal of Physiology, 204, 283-298.

Campbell, F. W., Cooper, G. F. \& Enroth-Cugell, C. (1969b). The spatial selectivity of the visual cells of the cat. Journal of Physiology, 203, 223235.

Campbell, F. W., Cooper, G. F., Robson, J. G. \& Sachs, M. B. (1969c). The spatial selectivity of cells of the cat and the squirrel monkey. Journal of Physiology, 120-121.

Campbell, F. W. \& Maffei, L. (1970). Electrophysiological evidence for the existence of orientation and size detectors in the human visual system. Journal of Physiology, 207, 635-652.

Campbell, F. W. \& Robson, F. G. (1968). Application of the Fourier analysis to the visibility of gratings. Journal of Physiology, 197, 551-566.

Carrasco, M., Mclean, T. L., Katz, S. M. \& Frieder, K. S. (1998). Feature asymmetries in visual search: Effects of display duration, target eccentricity, orientation and spatial frequency. Vision Research, 38(3), 347-374.

Comsweet, T. N. (1970). Vision perception. New York: Academic Press.

de Lange, H. (1952). Experiments on flicker and some calculations on an electrical analogue of the foveal systems. Physica, 18, 935-950.

de Lange, H. (1957). Attenuation characteristicsand phase-shiftcharacteristicsof thehuman fovea-cortex systemsin relation toflickerfusion phenomena. Technical University, Delft.

De Valois, R. L \& De Valois, K. K. (1988). Spatial vision. New York: Oxford University Press.

Dorais, A. \& Sagi, D. (1997). Contrast masking effects change with putative. Vision Research, 37, 1725-1733.

Garcia, J. A., Nieves, J. L, Valero, E. \& Romero, J. (2000). Stochastic independence of color-vision mechanisms confirmed by subthreshold summation paradigm. Journal of theOptical Society of America and Optical Image ScienceVision, 17(8), 1485-1488.

Georgeson, M. A. \& Sullivan, G. D. (1975). Contrast constancy: Deblurring in human vision by spatial frequency channels. Journal of Physiology, 252, 627-656.

Gilinski, A. S. (1968). Orientation-specific effects of patterns of adapting light on visual acuity. Journal of theOptical Society of America, 58, 13-18.

Ginsburg, A. P., Cannon, M. W. \& Nelson, M. A. (1980). Suprathreshold processing of complex visual stimuli: Evidence for linearity in contrast perception. Science, 208, 619-621.

Graham, N. (1972). Spatial frequency channels in the human visual system: Effects of luminance and pattem drift rate. Vision Research, 12, 53-63.

Graham, N. (1980). Spatial frequency channels in human vision: Detecting edges without edge detectors. Em C. S. Hartis (Org.), Visual coding and adaptability (pp. 215-262). Hillsdale, New York: Lawrence Erlbaum.

Graham, N. \& Nachmias, J. (1971). Detection of grating patterns containing two spatial frequencies: A comparison of single-channel and multiple channel models. Vision Research, 11, 251-259.

Greenlee, M. W. \& Magnussen, S. (1998). Limited-capacity mechanisms of visual discrimination. Vision Research, 38(3), 375-385.

Helmholtz, H. V. (1877). On the sensations of tone. New York: Dover.

Howard, C. M., Tregear, S. J. \& Werner, S. J. (2000). Time courseof early mesopic adaptation to luminance decrements and recovery of spatial resolution. Vision Research, 40(22), 3059-64.

Humanski, R. A. \& Wilson, H. R. (1993). Spatial-frequency adaptation: Evidence for a multiple-channel model of short-wavelength-sensitivecone spatial vision. Vision Research, 5, 665-675.

Issa, N. P., Trepel, C. \& Stryker, M. P. (2000). Spatial frequency maps in cat visual cortex. Journal of Neuroscience, 20(22), 8504-8514.

Ives, H. E. (1922). Critical frequency relations in scotopic vision. Journal of theOptical Society of America, 6, 254-268.
Jones, R. M. \& Tulunay-Keesey, U. (1975). Local retinal adaptation and spatial frequency channels. Vision Research, 15, 1239-1244.

Julesz, B. (1980). Spatial-frequency channels in one-, two-, and three-dimensional vision: Variations on an auditory theme by Bekesy. Em C. S. Hamis (Org.), Visual coding and adaptability (pp.263-316). Hillsdale, New Jersey: Lawrence Erlbaum.

King-Smith, P. E. \& Kulikowski, J. J. (1975). The detection of gratings by independent activation of line detectors. Journal of Physiology, 245, 237-271.

Kortum, P. T. \& Geisler, W. S. (1995). Adaptation mechanisms in spatial vision - II. Flash thresholds and background adaptation. Vision Research, 35, 1595-1602.

Kulikowski, J. J. \& Gorea, A. (1978). Complete adaptation to patterned stimuli: A necessary and sufficient condition for Weber's law for contrast. Vision Research, 18, 1223-1227.

Kulikowski, J. J. \& King-Smith, P. E. (1973). Spatial arrangement of line, edge and grating detectors relealed by sub-threshold summation. Vision Research, 13, 1455-1478.

Legge, G. E. (1978). Space domain properties of a spatial frequency channel in human vision. Vision Research, 18, 959-969.

Legge, G. E. (1981). A power law for contrast discrimination. Vision Research, 21, 457-469.

Maffei, L \& Fiorentini, A. (1973). The visual cortex as a spatial frequency analyzer. Vision Research, 13, 1255-1267.

Maffei, L., Fiorentini, A. \& Bisti, S. (1973). Neural correlates of perceptual adaptation to gratings. Science, 182, 1036-1038.

Mansfield, J. S. \& Parker, A. J. (1993). An orientation-tuned component in the contrast masking of stereopsis. Vision Research, 33, 1535-1544.

Meese, T. S. \& Georgeson, M. A. (1996). Spatial filter combination in human pattern vision: Channel interactions revealed by adaptation. Perception, 25, 255-277.

Menees, S. M. (1998). The effect of spatial frequency adaptation on the latency of spatial contrast detection. Vision Research, 38, 3933-3942.

Mussap, A. J. \& Levi, D. M. (1997). Vemier acuity with plaid masks: The role of oriented filters in Vernier acuity. Vision Research, 37, 1325-1340.

Nachmias, J., Sansbury, R, Vassilev, A. \& Weber, A. (1973). Adaptation to square-wave gratings: In search of the elusive thind harmonic. Vision Research, 13, 1335-1342.

Ohm, G. S. (1843). Ueber die Definition des Tones, nebst daran geknupfter Theorie der Sirene und ahnlicher tonbildener Vorrichtungen. Annual Physiology Chemistry, 135, 497-565.

Olds, E. S. \& Engel, S. A. (1998). Linearity across spatial frequency in object recognition. Vision Research, 38, 2109-2118.

Polat, U. \& Sagi, D. (1993). Lateral interactions between spatial channels: Suppression and facilitation revealed by lateral masking experiments. Vision Research, 33, 993-999.

Sachs, M. B., Nachmias, J. \& Robson, J. G. (1971). Spatial frequency channels in human vision. Journal of the Optical Society of America, 61, 1176-1186.

Santos, N. A. (1996). Sistema visual humano: Filtragem defreqüênciasradiais moduladaspor perfisde Bessel j0,j1, j2, j4, j8 ej16. Dissertação de Mestrado não-publicada, Curso de Pós-Graduação em Neurociências e Comportamento, Instituto de Psicologia, Universidade de São Paulo. São Paulo, SP.

Santos, N. A. (1999). Sistema visual humano: Curvasdesensibilidadeefiltragem de freqüênciasangulares, radiaiseradial/angularesacopladas. Tese de Doutorado não-publicada, Curso de Pós-Graduação em Neurociências e Comportamento, Instituto de Psicologia, Universidade de São Paulo. São Paulo, SP.

Santos, N. A. \& Simas, M. L B. (2001). Percepção e processamento visual da forma: Discutindo modelos teónicos atuais. Psicologia: Reflexão \&Crítica, 14(1), 151-160.

Schade, O. H. (1948). Electro-optical characteristics of television systems 1. Characteristics of vision and visual systems. RCA Review, 9, 5-37.

Schade, O. H. (1956). Optical and photoelectric analog of the eye. Journal of theOptical Society of America, 46, 721-739. 
Sekuler, R. (1974). Spatial vision. Annual Review of Psychology, 25, 195-232.

Selwyn, E. W. H. (1948). The photographic and visual resolving power of lenses. 1. Visual resolving power. Photographic Journal, 88B, 6-12.

Simas, M. L B. (1985). Linearityand domain invariancein thevisual system. Tese de Doutorado não-publicada, Queen's University at Kingston. Ontário, Canada.

Simas, M. L. B. \& Dodwell, P. C. (1990). Angular frequency filtering: A basis for pattem decomposition. Spatial Vision, 5, 59-74.

Simas, M. L B., Frutuoso, J. T. \& Vieira, F. M. (1992). Inhibitory sidebands in multiple angular filters in the human visual system. Brazilian Journal of Medical Biological Research, 25, 919-923.

Simas, M. L B. \& Santos, N. A. (1998). Human frequency response functions of harmonic 2, 4, 8, and 16 cycle angular frequency filters. Trabalho apresentado no Internacional Symposium on Computer Graphics, Image Processing and Vision, IEEEComputer Science (pp. 312-319).

Smith, R. A. (1977). Spatial-frequency adaptation and afterimages. Perception, 6, 153-160.

Snowden, R. J. \& Hammett, S. T. (1996). Spatial frequency adaptation: Threshold elevation and perceived contrast. Vision Research, 36(12), 1997-1809.

Sperling, G. (1964). Linear theory and the psychophysics of flicker. Documenta Ophtalmologica, 18, 3-15.

Stromeyer, C. F. \& Julesz, B. (1972). Spatial-frequency masking in vision: Critical bands and spread of masking. Journal of the Optical Society of America, 62, 1221-1232.
Stromeyer, C. F., Klein, S., Dawson, B. M. \& Spillmann, L. (1982). Low spatial-frequency channels in human vision: Adaptation and masking. Vision Research, 22, 225-233.

Tolhurst, D. J. \& Barfield, L P. (1978). Interactions between spatial frequency channels. Vision Research, 18, 851-858.

Wainwright, M. J. (1999). Visual adaptation as optimal information transmission. Vision Research, 39(23), 3960-74.

Wilson, H. R., McFarlane, D. H. \& Phillips, G. C. (1983). Spatial frequency tuning of orientation selective units estimated by oblique masking. Vision Research, 23, 873-882.

Wilson, H. R., Nagy, S. E., Mets, M. B. \& Perrera, V. P. (1988). Spatial frequency and orientation tunning of spatial visual mechanisms in human albinos. Vision Research, 28, 991-999.

Wilson, H. R. \& Wilkinson, F. (1997). Evolving concepts of spatial channels in vision: From independence to nonlinear interactions. Perception, $26,939-960$

Yang, J. \& Stevenson, S. B. (1999). Post-retinal processing of background luminance. Vision Research, 39(24), 4045-4051.

\section{Sobre os autores}

Natanael Antonio dos Santosé Doutorem Psicologia, na área de Neurociênciase Comportamento, pelo Instituto de Psicologia da Universidade de São Paulo. Pesquisador do CNPq, Bolsa DCR, vinculado ao Departamento de Psicologia da UFPE.

Maria Lúcia de Bustamante Simas é Doutora em Psicologia pela Queen's University, Ontario, Canadá. Professora Adjunta do Departamento de Psicologia da UFPE.

Recebido: 19/09/2001

Revisado: 14/02/2002

Aceite Final: 14/05/2002 